\title{
Classical Approach for the Fusion Reaction of Deuterium-Tritium Using 2-D Velocity Verlet Method Dani Irawan ${ }^{*}$, Sparisoma Viridi ${ }^{2}$, Rizal Kurniadi ${ }^{2}$, Abdul Waris ${ }^{2}$ \\ ${ }^{1}$ Chalmers University of Technology, Gothenburg 41296, Sweden \\ ${ }^{2}$ Institut Teknologi Bandung, Jalan Ganeca No. 10, Bandung 40132, Indonesia
}

Received: 23 June 2015, Revised: 5 Aughust 2015, Accepted: 2 December 2015

\begin{abstract}
Fusion reaction simulation of two types of nucleon: proton and neutron, is reported in this paper. The interactions between these nucleons are assumed to be only the nuclear force and electrostatic. The modeling is done in a classical approach where molecules formed by the nucleons are being collided with each other. In the model, some parameters were configured to see how these parameters affect the simulation. Some interesting results is that we can find a stable Helium-5 and a neutron-neutron pair as the product of the collision which should not have been possible due to the the quark interactions inside them. This leads us to a conclusion that we need to add a force-model for quark interaction. We do this by using an electrostatic-like force. However using this model, we may end up to instability of the tritium. In this paper we also report a configuration where the neutron-pair is unstable while tritium is stable.
\end{abstract}

Keywords: Molecular dynamics, fusion reaction

\section{INTRODUCTION}

Fusion reaction of deuterium with tritium producing helium-4 ${ }^{[1]}$ is a very common reaction. A model of this reaction using fifth order Gear method has been reported and shows a wide variety of reaction product depending on the configuration of the model ${ }^{[2]}$. One of the product observed in the report is a neutron-pair which even though possible to create ${ }^{[3,4]}$, but it is very uncommon and unstable $^{[5]}$.

A same reaction model with different method has been conducted and will be reported in this paper. The method used here is the velocity Verlet method. We then compare the new model with the previous model ${ }^{[2]}$. Then another simulations are performed to study the stability of the neutron pair and tritium in different configuration.

\section{SIMULATION}

In the simulation, we used 4 different force model: nuclear binding force $B_{i j}$, nuclear repulsion force $R_{i j}$, electrostatic force $Q_{i j}$, and quark interaction force $S_{i j}$. The nuclear binding force

Corresponding author

E-mail address: irawan_dani@yahoo.com represent the strong interaction, one of the 4 fundamental interactions. Nuclear repulsion force is a representation of the fact that two nucleons cannot have a same position. Electrostatic force is used because we have protons. The quark interaction force is introduced to prevent the forming of neutron-pair.

The first three force models are calculated with the sama equation as in previous model ${ }^{[2]}$ :

$$
\begin{gathered}
\vec{B}_{i j}=\hat{r}_{i j}\left(k_{b} \frac{b_{i} b_{j}}{r_{i j}^{2}}\right), r_{i j}<r_{b}, \\
\vec{R}_{i j}=k_{r} \hat{r}_{i j}\left(\max \left(0, \frac{1}{2}\left(d_{i}+d_{j}\right)-r_{i j}\right)\right), \\
\vec{Q}_{i j}=\hat{r}_{i j}\left(k_{q} \frac{q_{i} q_{j}}{r_{i j}{ }^{2}}\right),
\end{gathered}
$$

$r_{b}$ is the cut-off distance for the strong interaction, $r_{i j}$ is the scalar distance between particle $i$ and $j$, and $\hat{r}_{i j}$ is the normalized displacement vector.

Quark interaction basically is same as electrostatic interaction. An up-quark has $a+2 / 3 \mathrm{e}$ charge, while a down-quark has $-1 / 3$ echarge. Proton consists of 2 up-quark and 1 down-quark resulting to a $+e$ net charge. Neutron consists of 2 down- 
quark and 1 up-quark resulting in a net charge of 0 , but internally it is a configuration of these three quarks ${ }^{[6]}$. Quark structure for proton and neutron make it possible to have a several stability modes ${ }^{[7]}$, We assume that this modes results in an averaged mode-constant $k_{s}$ therefore we can calculate the force from quark interaction with a similar equation with the electrostatic force.

$$
\vec{S}_{i j}=\hat{r}_{i j}\left(k_{s} \frac{s_{i} s_{j}}{r_{i j}{ }^{2}}\right),
$$

We set $s=-1$ for neutron and 1 for proton. With these values, the interaction will add a repulsive force to the neutron-pair while a proton-neutron pair will still bind. Tritium binding however, cannot be predicted easily because it is practically a multibody system. as follows:

The algorithm for velocity Verlet method is

$$
\begin{aligned}
& \vec{x}_{i}(t+\Delta t)=\vec{x}_{i}(t)+\vec{v}_{i}(t) \Delta t+\frac{1}{2} \vec{a}_{i}(t) \Delta t^{2}, \\
& \vec{v}_{i}(t+\Delta t)=\vec{v}_{i}(t)+\frac{\left(\vec{a}_{i}(t)+\vec{a}_{i}(t+\Delta t)\right)}{2} \Delta t,
\end{aligned}
$$

$x_{\mathrm{i}}, v_{i}$, and $a_{i}$ are position, velocity, and acceleration of particle $i^{[8]}$.

The initial configuration of the simulation is shown in figure 1 .

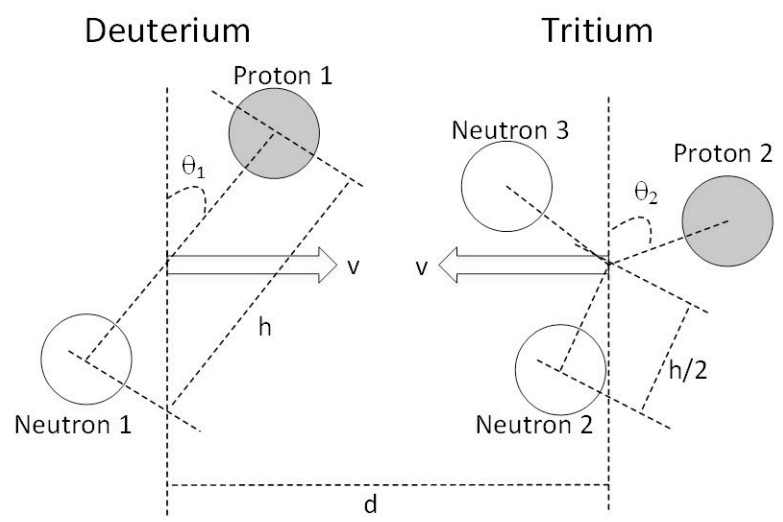

Fig. 1. Initial configuration of the reaction model

Furthermore, to test stability of neutron pair and tritium, a similar configuration is also set but changing the proton in deuterium into a neutron. In this configuration, the separation $d$ is set far away such that the interaction between the two clusters are negligible.

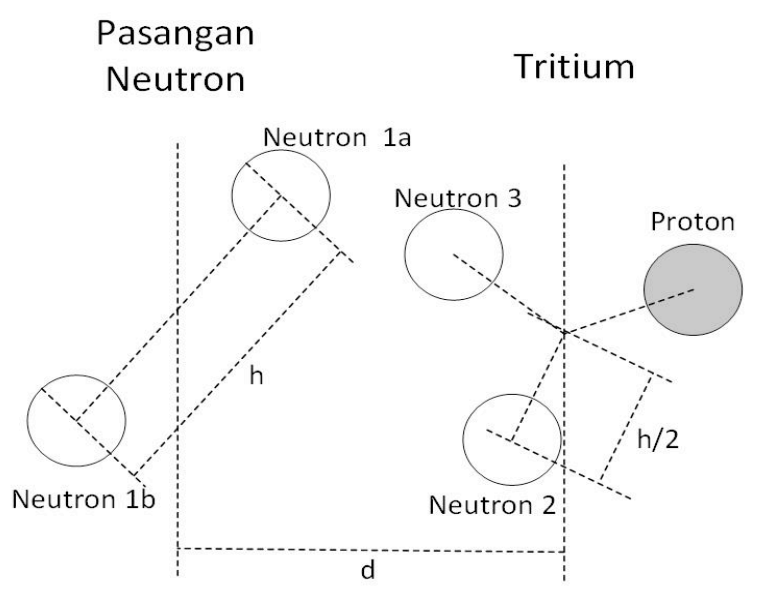

Fig. 2. Initial configuration for neutron-pair repulsion test

\section{RESULTS AND DISCUSSION}

Model parameters are set to be same as a previous mode ${ }^{[1]}$ to compare the methods. The simulation result for velocity Verlet method is shown in figure 3 .

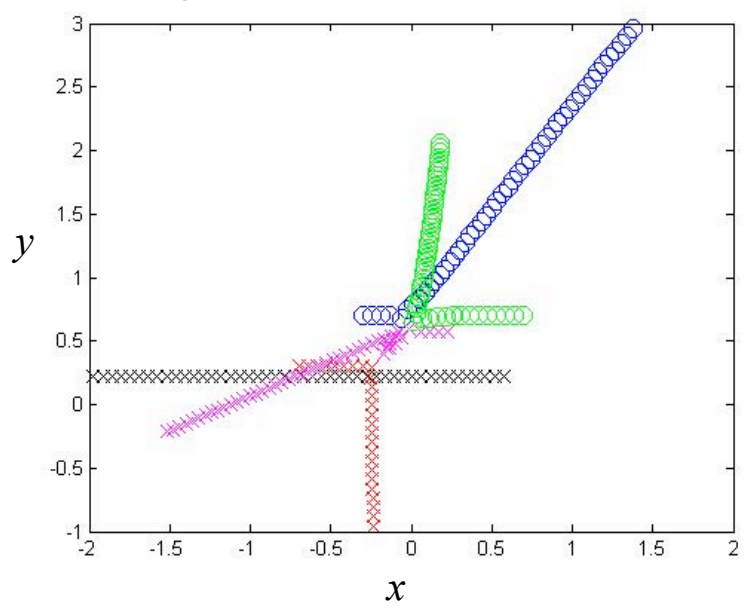

Fig. 3. Particle trajectory. Parameter: $m=0.1, q=0.1, d=1, b=1$, $k_{b}=0.2, \quad r_{b}=0.2, \quad k_{r}=10^{5}, k q=0.01, \quad \theta_{l}=\pi / 4, \quad \mathrm{q} \theta_{2}=\pi / 4$, $h=0.4 \sqrt{2}, v=\sqrt{2}$.

With the configuration shown in figure 3 caption, the products in previous model are 2 deuteriums and 1 neutron while in the velocity Verlet model no particle is binding with each other. This implies that for the velocity Verlet method, we may need a higher magnitude of binding force or the cut-off distance is too short. We further test the model by modifying the parameters. Another result is presented in figure 4. 


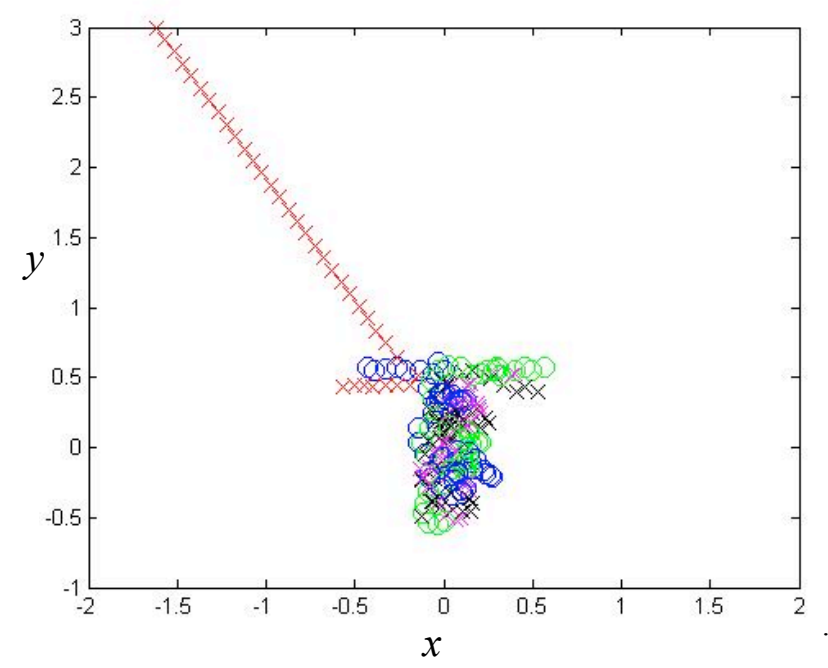

Fig. 4. Particle trajectory. Parameter: $m=0.1, q=0.1, d=1$, $b=1, k_{b}=0.1, r_{b}=0.4 \sqrt{2}, k_{r}=10^{5}, k_{q}=0.01, \theta_{l}=\pi / 4$, $\theta_{2}=\pi / 4, h=0.2, v=\sqrt{2}$.

Using the configuration presented in figure 4 , the reaction product is now helium- 4 which is actually the most common product in the reaction. However, when we apply this configuration without the quark interaction term to the neutron-pair repulsion test, the neutron-pair is stable.

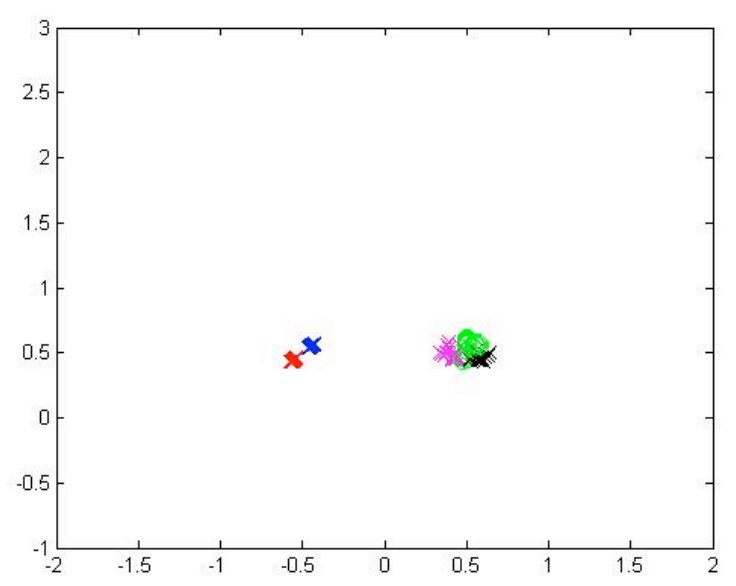

Fig 5. Left: stable neutron-pair, right: stable tritium. Parameter: $m=0.1, q=0.1, d=1, b=1, k_{b}=0.1, r_{b}=0.4 \sqrt{2}$, $k_{r}=10^{5}, k_{q}=0.01, h=0.2, t_{\max }=1, \Delta t=10^{-6}$.

Adding the quark interacton term, in some configuration, the neutron pair will become unstable. But, tritium stability cannot be ensured as can be seen in figure 6 and 7 .

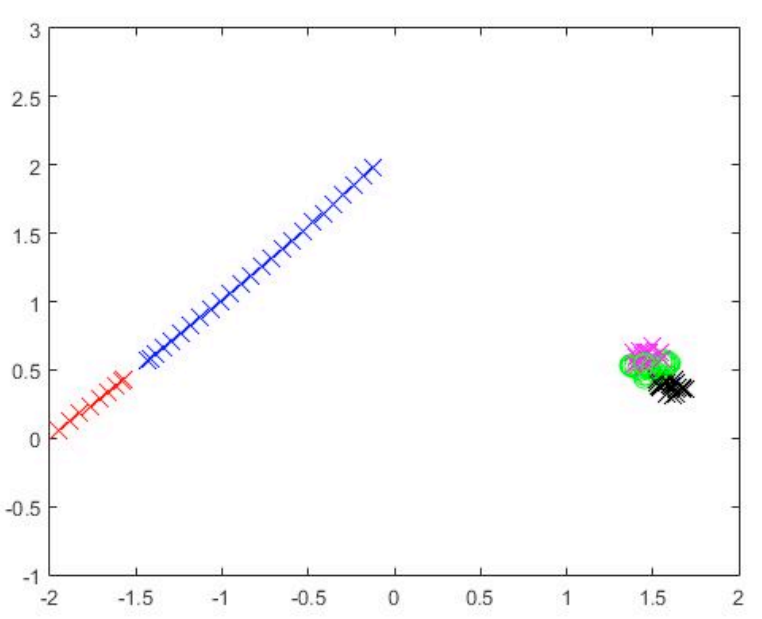

Fig. 6. Left: unstable neutron-pair, right: stable tritium $m=0.1$, $q=0.1, d=3, b=1, k_{b}=0.04, k_{s}=0.14, r_{b}=0.4 \sqrt{2}, k_{r}=10^{5}, k_{q}=0.01$, $h=0.2, t_{\max }=1, \Delta t=10^{-6}$

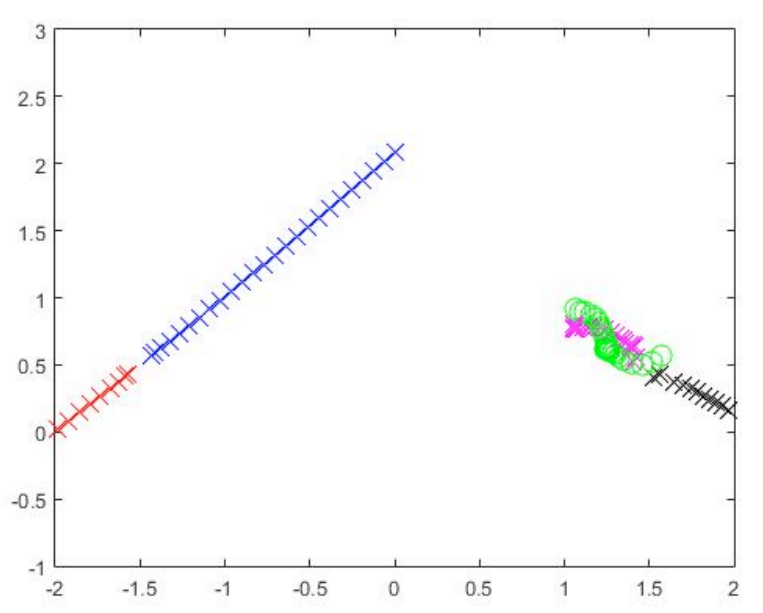

Fig 7. Left: unstable neutron-pair, right: unstable tritium (the black nucleon escapes from binding). $m=0.1, q=0.1, d=3, b=1$, $k_{b}=0.02, \quad k_{s}=0.14, \quad r_{b}=0.4 \sqrt{2}, \quad k_{r}=10^{5}, k_{q}=0.01, \quad h=0.2$, $t_{\max }=1, \Delta t=10^{-6}$

Neutron-pair and tritium stability depends on the value of $k_{s}$ and $k_{b}$. Figure 8 shows a parameter space for the stability. 


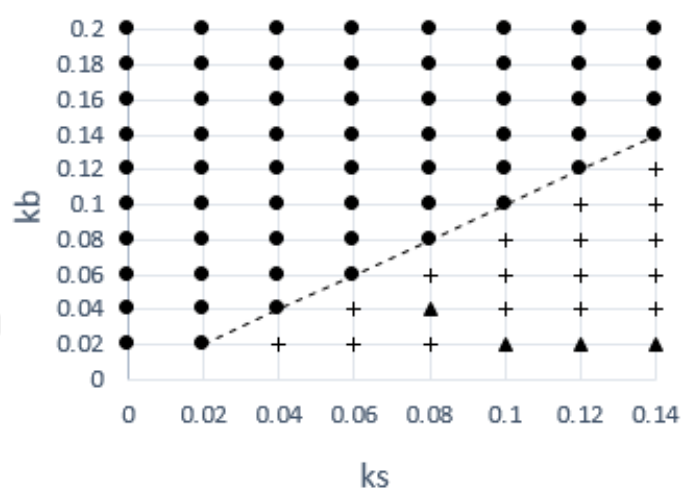

- N-N Stable, Tritium Stable

- N-N Unstable, Tritium Unstable

$+\mathrm{N}-\mathrm{N}$ Unstable, Tritium Stable

$\cdots \cdot \cdots \cdot k b$

Fig. 8. Stability chart of neutron-pair and tritium in a $k_{s}$ and $k_{b}$ space.

In figure 8 , we can see that neutron-pair will be unstable when $k_{s}>k_{b}$. On the other hand, we cannot draw a conclusion yet for tritium stability.

\section{CONCLUSION}

The simulations shows that the neutron-pair is observed when using a velocity Verlet method. The binding of the neutron pair can be cut by adding a quark interaction term as a classical interaction on the model with a constraint $k_{s}>k_{b}$. In this situation, tritium binding may be affected and thus needs further investigation.

\section{ACKNOWLEDGMENT}

The publication of this report is supported by Program Riset ITB 2015 Batch II with contract number: 1763/I1.B04.1/KU/2015.

\section{REFERENCES}

[1] J. Kenneth Shultis dan Richard E. Faw, "Fundamental of Nuclear Science and Engineering", Marcel-Dekker, Inc., chapter 6.7: Fusion Reactions.

[2] Sparisoma Viridi, Rizal Kurniadi, Abdul Waris, dan Yudha Satya Perkasa, "A Classical
Approach in Simple Nuclear Fusion Reaction ${ }_{1} \mathrm{H}^{2}+{ }_{1} \mathrm{H}^{3}$ using Two-Dimension Granular Molecular Dynamics Model", The 3rd International Conference on Advances in Nuclear Science and Engineering-2011, edited by Z. Su'ud et al., AIP Conference Proceedings 1448, American Institute of Physics, Melville, NY, 2012, pp. 170-176, doi: $10.1063 / 1.4725452$

[3] Michael Schirber, "Nuclei Emit Paired-up Neutrons", Physics 5: 30, doi:10.1103/Physics.5.30.

[4] A. Spyrou, Z. Kohley, T. Baumann, D. Bazin, B.A. Brown G. Christian, P. A. DeYoung, J.E. Finck, N. Frank, E. Lunderberg, S. Mosby, W. A. Peters, A. Schiller, J.K. Smith, J. Snyder, M.J. Strongman, M. Thoennessen, dan A. Volya, "First Observation of Ground State Dineutron Decay: ${ }^{16} \mathrm{Be} "$, Physical Review Letters 108, 102501, doi: 10.1103/PhysRevLett.108.102501

[5] J. MacDonald dan D.J. Mullan, "Big bang nucleosynthesis: The strong nuclear force meets the weak anthropic principle", Physical Review D 80, 043507, doi: 10.1103/PhysRevD.80.043507

[6] David Griffiths, "Introduction to Elementary Particles, Second, Revised Edition", Wiley VCH Verlag GmbH \& Co. KgaA, Weinheim, pp. XIV, 39

[7] Jerry Montgomery dan Rondo Jeffery, "Neutron-Neutron, Proton-Proton, verses Proton-Neutron (Deuteron) Enigma Explained", URI http://www.unclear2nuclear.com/np.php [Accessed: 22 Juni 2015]

[8] William C. Swope, H.C. Andersen, P.H. Berens, dan K.R. Wilson, "A computer simulation method for the calculation of equilibrium constants for the formation of physical clusters of molecules: Application to small water clusters", The Journal of Chemical Physics 76 (1), doi:10.1063/1.442716. 